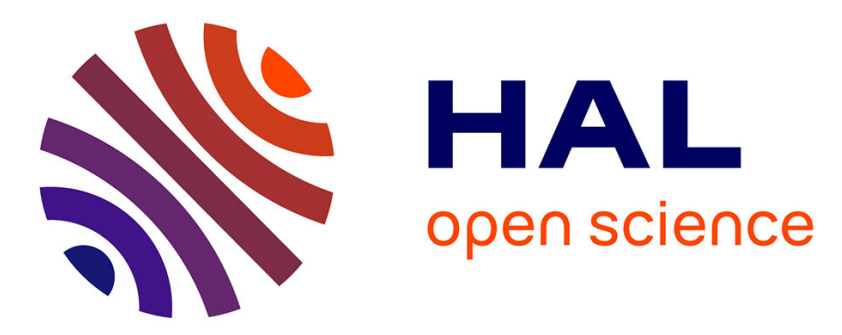

\title{
Un modèle non-linéaire de prolifération cellulaire : extinction des cellules et invariance
}

\author{
Mostafa Adimy, Fabien Crauste
}

\section{To cite this version:}

Mostafa Adimy, Fabien Crauste. Un modèle non-linéaire de prolifération cellulaire: extinction des cellules et invariance. Comptes Rendus. Mathématique, 2003, 336 (7), pp.559-564. 10.1016/S1631073X(03)00125-0 . hal-00375916

\section{HAL Id: hal-00375916 https://hal.science/hal-00375916}

Submitted on 16 Apr 2009

HAL is a multi-disciplinary open access archive for the deposit and dissemination of scientific research documents, whether they are published or not. The documents may come from teaching and research institutions in France or abroad, or from public or private research centers.
L'archive ouverte pluridisciplinaire HAL, est destinée au dépôt et à la diffusion de documents scientifiques de niveau recherche, publiés ou non, émanant des établissements d'enseignement et de recherche français ou étrangers, des laboratoires publics ou privés. 


\title{
Un modèle non-linéaire de prolifération cellulaire : extinction des cellules et invariance
}

\author{
Mostafa Adimy* et Fabien Crauste ${ }^{\dagger}$
}

Année 2002

\begin{abstract}
Laboratoire de Mathématiques Appliquées Université de Pau et des Pays de l'Adour Avenue de l'université, 64000 Pau, France
\end{abstract}

\begin{abstract}
Résumé
Dans ce travail, nous étudions un système non-linéaire structuré en âge et maturité décrivant la production des cellules sanguines dans la moëlle osseuse. Après intégration du modèle, nous obtenons une équation aux dérivées partielles du premier ordre faisant apparaître un retard distribué en temps et une dépendence non-locale dans la variable maturité. Nous prouvons que l'unicité des solutions ne dépend que des cellules de petites maturités (cellules souches) et nous donnons un résultat d'invariance.
\end{abstract}

\section{Introduction}

Nous nous intéressons dans ce travail à un modèle mathématique non-linéaire décrivant une population cellulaire : la production des cellules sanguines dans la moëlle osseuse, à partir des cellules souches. Ce modèle est décrit par un système de deux équations aux dérivées partielles structurées en âge et maturité.

Les modèles de populations biologiques structurés en âge et maturité sont apparus vers la fin des années 60. Les premiers à les avoir étudiés sont Keyfitz [4] (1968), Pollard 8] (1973), Henry [3] (1976) et Mackey [5] (1978). Le modèle de Mackey, dans lequel la population cellulaire est supposée uniquement proliférer, a été étudié plus amplement à partir de 1990, notamment numériquement par Mackey et Rey dans [10] (1992) et 11] (1993). En 1994, Mackey et Rudnicki [7] ont complété ce modèle en tenant compte d'une phase de repos en plus de la phase de prolifération. Ils obtiennent les équations sur lesquelles nous allons travailler et qui sont présentées en 2.1 et 2.2. Dans la phase de prolifération, dont la durée est limitée, les cellules synthétisent de l'ADN puis se divisent, donnant naissance à deux cellules filles. Chacune des cellules filles entre ensuite immédiatement dans la phase de repos. Elle

\footnotetext{
*Mél : mostafa.adimy@univ-pau.fr

†Mél : fabien.crauste@univ-pau.fr
} 
peut y demeurer toute sa vie et y mourir de vieillesse ou bien être introduite dans la phase de prolifération à un certain moment. Nous étudirons ici le cas non-linéaire où le taux de réintroduction dépend de la population totale de cellules au repos.

Mackey et Rudnicki montrent que le système d'équations aux dérivées partielles qu'ils ont obtenu devient, après intégration, un système d'équations aux dérivées partielles à retard. Dyson, Villella-Bressan et Webb en 1996 [2] et Adimy et Pujo-Menjouet en 2001 [1] ont montré que la population de cellules de petites maturités influence le comportement de la population totale, dans le cas où les cellules sont supposées se diviser toujours au bout du même temps.

Nous nous proposons d'étudier le modèle proposé par Mackey et Rudnicki en 1994 [7] en tenant compte d'une remarque faite par Mackey et Rey dans [11 concernant la division cellulaire : nous supposerons que l'âge de la cytocinèse (le point de division cellulaire) est distribué selon une densité de support $[\underline{\tau}, \bar{\tau}]$, avec $0<\underline{\tau}<\bar{\tau}<+\infty$. Dans [11], Mackey et Rey n'ont étudié que numériquement ce modèle et sans tenir compte de la phase de repos. Dans les travaux de Mackey et Rudnicki [7], Dyson, Villella-Bressan et Webb [2] et Adimy et Pujo-Menjouet [1], les cellules proliférantes se divisaient toujours au bout du même temps fixe.

Enfin, rappelons que la production de cellules sanguines peut avoir deux comportements différents. Le premier correspond à une production normale de cellules et a lieu lorsque la population de cellules souches (c'est-à-dire les cellules de petites maturités) est strictement positive. Le deuxième mène à une production anormale de cellules et est le résultat de la destruction ou de l'absence de cellules souches dans le milieu originel : ce cas correspond à l'anémie aplasique, une maladie s'attaquant aux cellules souches.

En supposant que la phase de prolifération est suffisament longue, nous allons montrer que l'unicité des solutions dépend uniquement des cellules souches et nous en déduirons un résultat d'invariance soulignant l'influence des cellules de petites maturités sur le comportement de la population totale.

Notre travail est organisé de la façon suivante : dans la section 2, nous présentons le modèle biologique sur lequel est basé notre travail; dans la section 3, nous obtenons, par intégration, le système d'équations aux dérivées partielles décrivant l'évolution de la population; dans la section 4 , nous donnons une formulation intégrée du problème; dans la section 5. nous établissons un résultat d'unicité des solutions ne dépendant que des cellules de petites maturités et dans la section 6 nous donnons un résultat d'invariance.

\section{Présentation du modèle}

La vie d'une cellule débute avec la division d'une autre cellule, dite cellule mère, et se termine, généralement, lors de sa propre division; la cellule donne alors naissance à deux nouvelles cellules appelées cellules filles. Durant sa vie, chaque cellule suit le même chemin que celui pris par sa mère : il s'agit du cycle cellulaire. Le cycle cellulaire tel que nous l'entendons ici comprend deux phases : une phase de prolifération et une phase de repos.

La phase de prolifération est la phase active du développement cellulaire : elle est dédiée à la synthèse de l'ADN et à la division cellulaire. Elle s'achève lors de la cytocynèse : la division de la cellule proprement dite. Une cellule ne peut rester indéfiniment dans la phase de prolifération : si elle ne se divise pas, elle meurt avec un taux $\gamma$. 
Juste après leur naissance, les cellules filles entrent immédiatement dans la phase de repos, ou phase $G_{0}$ (de l'anglais Gap), qui est un stade quiescent du développement cellulaire : les cellules au repos sont inactives. Contrairement à ce qui se passe dans la phase de prolifération, les cellules peuvent rester toute leur vie dans la phase de repos. La plupart seront introduites à un certain moment dans la phase de prolifération et complèteront ainsi le cycle cellulaire, mais les cellules au repos peuvent aussi mourir, avec un taux $\delta$.

Une cellule sera caractérisée par son âge et sa maturité. La maturité désigne un aspect morphologique du développement de la cellule, au même titre que la taille ou l'âge; il s'agit d'une variable continue : dans l'évaluation de la maturité, on tient compte, par exemple, du niveau de synthèse d'ADN, d'ARN, de la présence de mitochondries, etc. La définition de la maturité est liée à la nature de la cellule. Nous supposerons que la maturité $m$ d'une cellule varie de $m=0$ à $m=1$.

Toutes les cellules proviennent des cellules embryonnaires issues des premiers jours du développement, appelées cellules souches (stem cells). Il s'agit de cellules indifférenciées et immatures (c'est-à-dire de petites maturités) et capables, d'une part, de se multiplier (et donc d'augmenter la quantité de cellules souches), et d'autre part, de donner naissance à des cellules différenciées (cellules du foie, cellules musculaires, cellules sanguines, ... ).

Il existe quatre types de cellules souches dont les cellules souches hématopoïétiques de la moëlle osseuse. Ce sont celles-ci qui nous intéressent car elles sont à l'origine de toutes les cellules sanguines : globules rouges, blancs, plaquettes, etc.

Mis à part leur importance dans le développement d'une population cellulaire, il faut noter que les cellules souches sont aussi à l'origine de certaines maladies, graves, dont l'anémie aplasique. Il s'agit d'une maladie rare et sérieuse caractérisée par l'absence de formation ou de division des cellules souches hématopoiétiques, entraînant une chute de la production des cellules sanguines.

Plusieurs facteurs expliquent l'apparition de cette maladie. Dans 50 à $65 \%$ des cas la nature de la maladie est idiopathique ou primitive, c'est-à-dire qu'elle ne comporte pas de cause connue. Dans les autres cas, elle est héréditaire ou due notamment à des expositions aux radiations nucléaires, à des toxines de l'environnement (insecticides, vapeurs de benzène, ... .) ou bien au traitement du cancer par radiothérapie, chimiothérapie ou d'autres médicaments.

\subsection{La phase de repos}

Nous désignons par $n(t, m, a)$ la densité de cellules dans la phase de repos à l'instant $t$, de maturité $m$ et d'âge $a$. Nous notons

$$
N(t, m)=\int_{0}^{\infty} n(t, m, a) d a
$$

le nombre total de cellules de maturité $m$ dans la phase de repos à l'instant $t$.

La densité de cellules $n(t, m, a)$ vérifie l'équation de conservation :

$$
\frac{\partial n}{\partial t}+\frac{\partial n}{\partial a}+\frac{\partial(V n)}{\partial m}=-(\delta+\beta) n
$$

où $\delta$ est le taux de mortalité, $\beta$ le taux de réintroduction des cellules dans la phase de prolifération et $V:[0,1] \rightarrow[0,+\infty)$ est la vitesse de maturation des cellules. 
$\delta=\delta(m)$ et $\beta=\beta(m, N(t, m))$ sont supposées positives et continues, et $\beta$ est supposée décroissante par rapport à $N(t, m) . V$ est de classe $C^{1}$ sur $[0,1]$ et vérifie $V(0)=0$ et $V(m)>0$ pour tout $m \in(0,1]$. De plus, nous supposerons que

$$
\int_{0}^{m} \frac{d s}{V(s)}=+\infty, \quad \text { pour tout } m \in(0,1] .
$$

Cela signifie qu'une cellule de maturité nulle ne peut jamais atteindre un état où sa maturité serait non nulle.

Exemple 2.1 Si $V(s) \underset{s \rightarrow 0}{\sim} \alpha s^{p}$, pour tout $s \in[0,1]$, avec $\alpha>0$ et $p \geq 1$, alors $\int_{0}^{m} \frac{d s}{V(s)}$ est une intégrale de Riemann divergente pour tout $m \in(0,1]$.

$n$ satisfait de plus la condition initiale :

$$
n(0, m, a)=\Upsilon(m, a), \quad \text { pour tout }(m, a) \in[0,1] \times[0,+\infty),
$$

où $\Upsilon$ est une fonction suffisament régulière vérifiant $\lim _{a \rightarrow \infty} \Upsilon(m, a)=0$.

\subsection{La phase de prolifération}

Dans la phase de prolifération, l'âge d'une cellule varie de 0 à $\bar{\tau}$ où $\bar{\tau}$ est la durée maximale de cette phase : parvenue à l'âge $\bar{\tau}$ une cellule doit s'être divisée sinon elle meurt. Cela entraîne qu'une cellule ne peut rester indéfiniment dans la phase de prolifération.

Soit $p(t, m, a)$ la densité de cellules de maturité $m$ et d'âge $a$ dans la phase de prolifération à l'instant $t$. Nous notons

$$
P(t, m)=\int_{0}^{\bar{\tau}} p(t, m, a) d a,
$$

le nombre total de cellules de maturité $m$ dans la phase de prolifération à l'instant $t$.

Soit $\gamma$ une fonction de la maturité $m$, positive et continue, représentant le taux de mortalité des cellules dans la phase de prolifération.

$p(t, m, a)$ vérifie l'équation de conservation :

$$
\frac{\partial p}{\partial t}+\frac{\partial p}{\partial a}+\frac{\partial(V p)}{\partial m}=-\gamma p
$$

où $V$ représente la vitesse de maturation des cellules dans cette phase, définie en 2.1. Nous supposons que la vitesse de maturation des cellules est la même dans les deux phases.

$p$ vérifie en plus la condition initiale :

$$
p(0, m, a)=\Gamma(m, a), \quad \text { pour tout }(m, a) \in[0,1] \times[0, \bar{\tau}],
$$

où $\Gamma$ est une fonction suffisament régulière, définie sur $[0,1] \times[0, \bar{\tau}]$.

Pour être bien posé, le système d'équations (10)-(3) doit être muni de conditions aux bords, traduisant le comportement des cellules lors des changements de phases. 


\subsection{Conditions aux bords}

En accord avec les remarques de Mackey et Rey (11]), nous supposerons que le point de cytocinèse des cellules proliférantes est distribué sur un intervalle $[\underline{\tau}, \bar{\tau}]$, où $0<\underline{\tau}<\bar{\tau}<+\infty$, selon une densité $a \mapsto k(m, a) . \bar{\tau}$ représente la durée maximale de la phase de prolifération tandis que $\underline{\tau}$ est choisi strictement positif car les cellules ne se divisent immédiatement après être entrées en phase de prolifération que dans le cas de certaines maladies qui ne nous intéressent pas ici.

Nous notons $g(m)$ la maturité d'une cellule fille à sa naissance, lorsque la maturité de la cellule mère valait $m$. La fonction $g:[0,1] \rightarrow[0,1]$ est supposée continue, strictement croissante sur $[0,1]$, de classe $\mathcal{C}^{1}$ sur $[0,1)$ et telle que $g(m)<m$ pour $m \in(0,1)$. $g$ est alors inversible et nous prolongeons $g^{-1}$ en posant $g^{-1}(m)=1$ si $m>g(1)$.

L'application $k$ est telle que $m \mapsto k(m, a)$ est la proportion de cellules mères d'âge $a$ dans la phase de prolifération pouvant donner après division une cellule fille de maturité $m$. $k$ est supposée positive et continue sur $[0,1] \times[\underline{\tau}, \bar{\tau}]$, et telle que $k(m, a)=0$ si $m \geq g(1)$. Ceci par définition de la fonction $g$ : la maturité d'une cellule mère ne pouvant être supérieure à 1 , celle d'une cellule fille ne peut excéder la valeur $g(1)$. Nous considèrerons donc, dans toute la suite, que la maturité d'une cellule fille est comprise entre 0 et $g(1)$.

La première condition au bord est alors donnée par

$$
n(t, m, 0)=2 \int_{\underline{\tau}}^{\bar{\tau}} k(m, a) p\left(t, g^{-1}(m), a\right) d a .
$$

Elle signifie qu'une cellule de maturité $m$ peut provenir d'un certain nombre de cellules mères, d'âges différents, contrairement aux modèles précedemment étudiés par Mackey et Rudnicki [7], Dyson, Villella-Bressan et Webb [2] et Adimy et Pujo-Menjouet [1], où la division survenait toujours au bout d'un temps fixe (la fin de la phase de prolifération).

La seconde condition aux limites est

$$
p(t, m, 0)=\int_{0}^{\infty} \beta(m, N(t, m)) n(t, m, a) d a=\beta(m, N(t, m)) N(t, m) .
$$

Elle représente le flux de la population de cellules, de la phase de repos vers la phase de prolifération.

Nous considérons le flot $\pi_{s}:[0,1] \rightarrow[0,1]$, défini pour $s \leq 0$, solution de l'équation différentielle ordinaire :

$$
\left\{\begin{aligned}
\frac{d u}{d s}(s) & =V(u(s)), \quad s \leq 0 \\
u(0) & =m
\end{aligned}\right.
$$

qui représente l'évolution de la maturité des cellules pour atteindre une maturité $m$ à l'instant 0 à partir d'un temps $s \leq 0 . \pi_{s}$ vérifie $\pi_{0}(m)=m, \pi_{s}(0)=0$ et $\pi_{s}(m) \in(0,1]$ pour $s \leq 0$ et $m \in(0,1]$.

En fait, nous pouvons remarquer (cf. [9]) que $\pi_{s}$ peut s'écrire explicitement

$$
\pi_{s}(m)=h^{-1}\left(h(m) e^{s}\right), \quad \text { pour } m \in[0,1] \text { et } s \leq 0,
$$

où $h:[0,1] \rightarrow[0,1]$ est défini par

$$
h(m)= \begin{cases}\exp \left(-\int_{m}^{1} \frac{d s}{V(s)}\right), & \text { pour } m \in(0,1] \\ 0, & \text { pour } m=0\end{cases}
$$


Notons que $h$ est une fonction continue et strictement croissante, donc inversible.

Exemple 2.2 Si $V(m)=\alpha m$, pour tout $m \in[0,1]$, avec $\alpha>0$, alors

$$
h(m)=m^{1 / \alpha}, \quad \forall m \in[0,1] .
$$

Donc, pour tout $m \in[0,1]$,

$$
h^{-1}(m)=m^{\alpha}
$$

et

$$
\pi_{s}(m)=m e^{\alpha s}, \quad \text { pour } s \leq 0 .
$$

Nous obtenons les fonctions utilisées par Rey et Mackey dans [1] et [1] et Dyson, VillellaBressan et Webb dans [A].

\section{Equations du modèle}

Avant de déterminer les équations du modèle sur lesquelles nous allons travailler nous établissons un lemme qui nous sera utile.

Soit $\Delta$ l'application définie de $[0,+\infty) \times[0, g(1)]$ dans $\mathbb{R}^{+}$par

$$
\Delta(s, m)=\pi_{-s}\left(g^{-1}(m)\right)=h^{-1}\left(h\left(g^{-1}(m)\right) e^{-s}\right) .
$$

Lemme 3.1 $\Delta$ satisfaits les assertions suivantes :

(i) l'application $s \in[0,+\infty) \mapsto \Delta(s, m)$ est décroissante pour chaque $m \in[0, g(1)]$,

(ii) l'application $m \in[0, g(1)] \mapsto \Delta(s, m)$ est croissante pour chaque $s \geq 0$, donc, pour tout $s \geq 0$ et tout $m \in[0, g(1)]$,

$$
0=\Delta(s, 0) \leq \Delta(s, m) \leq \Delta(s, g(1))=h^{-1}\left(e^{-s}\right),
$$

(iii) pour tout $m \in(0, g(1)]$,

$$
\Delta(s, m)<m \quad \text { ssi } \quad s>\int_{m}^{g^{-1}(m)} \frac{d \theta}{V(\theta)},
$$

(iv) pour tous $\sigma, s \geq 0$, et tout $m \in[0, g(1)]$,

$$
\pi_{-\sigma}(\Delta(s, m))=\Delta(s+\sigma, m) .
$$

Preuve : Les points (i) et (ii) sont évidents par définition, les fonctions $h$ et $g$ étant croissantes sur $[0,1]$.

Pour montrer le point (iii), remarquons que $\int_{m}^{g^{-1}(m)} \frac{d \theta}{V(\theta)}=\ln \frac{h\left(g^{-1}(m)\right)}{h(m)}$ pour tout $m>0$. Alors nous avons, pour tout $m \in(0, g(1)]$ et tout $s \geq 0$,

$$
\begin{aligned}
\Delta(s, m)<m & \Leftrightarrow h\left(g^{-1}(m)\right) e^{-s}<h(m) \\
& \Leftrightarrow \quad-s<\ln \frac{h(m)}{h\left(g^{-1}(m)\right)} \\
& \Leftrightarrow s>\int_{m}^{g^{-1}(m)} \frac{d \theta}{V(\theta)} .
\end{aligned}
$$


D'où le résultat.

Montrons (iv). Par définition nous avons, pour tous $\sigma, s \geq 0$ et tout $m \in[0, g(1)]$,

$$
\begin{aligned}
\pi_{-\sigma}(\Delta(s, m)) & =h^{-1}\left(h\left(h^{-1}\left(h\left(g^{-1}(m)\right) e^{-s}\right)\right) e^{-\sigma}\right) \\
& =h^{-1}\left(h\left(g^{-1}(m)\right) e^{-(s+\sigma)}\right) \\
& =\Delta(s+\sigma, m) .
\end{aligned}
$$

Le lemme est ainsi démontré.

Nous utiliserons, dans toute la suite, les notations suivantes :

$$
\xi(m, t)=\exp \left\{-\int_{0}^{t} \gamma\left(\pi_{-s}(m)\right)+V^{\prime}\left(\pi_{-s}(m)\right) d s\right\},
$$

pour tout $m \in[0,1]$ et tout $t \geq 0$, et

$$
\zeta(m, a)=k(m, a) \xi\left(g^{-1}(m), a\right),
$$

pour tout $m \in[0,1]$ et tout $a \geq 0$.

Nous établissons le résultat suivant.

Proposition 3.1 Le nombre total, $N(t, m)$, de cellules de maturité $m$ dans la phase de repos, vérifie les équations suivantes:

(i) Si $0 \leq t \leq \underline{\tau}$ et $m \in[0, g(1)]$,

$$
\begin{aligned}
\frac{\partial}{\partial t} N(t, m) & +\frac{\partial}{\partial m}(V(m) N(t, m))=-(\delta(m)+\beta(m, N(t, m))) N(t, m) \\
& +2 \xi\left(g^{-1}(m), t\right) \int_{\underline{\tau}}^{\bar{\tau}} k(m, a) \Gamma(\Delta(t, m), a-t) d a .
\end{aligned}
$$

(ii) Si $\underline{\tau} \leq t \leq \bar{\tau}$ et $m \in[0, g(1)]$,

$$
\begin{gathered}
\frac{\partial}{\partial t} N(t, m)+\frac{\partial}{\partial m}(V(m) N(t, m))=-(\delta(m)+\beta(m, N(t, m))) N(t, m) \\
+2 \int_{\underline{\tau}}^{t} \zeta(m, a) \beta(\Delta(a, m), N(t-a, \Delta(a, m))) N(t-a, \Delta(a, m)) d a \\
+2 \xi\left(g^{-1}(m), t\right) \int_{t}^{\bar{\tau}} k(m, a) \Gamma(\Delta(t, m), a-t) d a .
\end{gathered}
$$

(iii) Si $t \geq \bar{\tau}$ et $m \in[0, g(1)]$,

$$
\begin{aligned}
& \frac{\partial}{\partial t} N(t, m)+\frac{\partial}{\partial m}(V(m) N(t, m))=-(\delta(m)+\beta(m, N(t, m))) N(t, m) \\
& \quad+2 \int_{\underline{\tau}}^{\bar{\tau}} \zeta(m, a) \beta(\Delta(a, m), N(t-a, \Delta(a, m))) N(t-a, \Delta(a, m)) d a .
\end{aligned}
$$


(iv) Si $t \geq 0$ et $m \in[g(1), 1]$,

$$
\frac{\partial}{\partial t} N(t, m)+\frac{\partial}{\partial m}(V(m) N(t, m))=-(\delta(m)+\beta(m, N(t, m))) N(t, m) .
$$

De même, la population totale, $P(t, m)$, de cellules de maturité $m$ dans la phase de prolifération vérifie les équations suivantes:

(v) Si $0 \leq t \leq \bar{\tau}$ et $m \in[0,1]$,

$$
\begin{aligned}
\frac{\partial}{\partial t} P(t, m)+\frac{\partial}{\partial m}(V(m) P(t, m)) & =-\gamma(m) P(t, m)+\beta(m, N(t, m)) N(t, m) \\
& -\Gamma\left(\pi_{-t}(m), \bar{\tau}-t\right) \xi(m, t) .
\end{aligned}
$$

(vi) Si $t \geq \bar{\tau}$ et $m \in[0,1]$,

$$
\begin{array}{r}
\frac{\partial}{\partial t} P(t, m)+\frac{\partial}{\partial m}(V(m) P(t, m))=-\gamma(m) P(t, m)+\beta(m, N(t, m)) N(t, m) \\
-\xi(m, \bar{\tau}) \beta\left(\pi_{-\bar{\tau}}(m), N\left(t-\bar{\tau}, \pi_{-\bar{\tau}}(m)\right)\right) N\left(t-\bar{\tau}, \pi_{-\bar{\tau}}(m)\right) .
\end{array}
$$

Preuve : Elle est basée sur la méthode des caractéristiques. Nous intégrons les équations (1) et (3) par rapport à l'âge :

$$
\begin{aligned}
\frac{\partial}{\partial t} P(t, m)+\frac{\partial}{\partial m}(V(m) P(t, m))= & -\gamma(m) P(t, m)+p(t, m, 0) \\
& -p(t, m, \bar{\tau}), \\
\frac{\partial}{\partial t} N(t, m)+\frac{\partial}{\partial m}(V(m) N(t, m))= & -(\delta(m)+\beta(m, N(t, m))) N(t, m) \\
& +n(t, m, 0),
\end{aligned}
$$

en ayant supposé que $\lim _{a \rightarrow \infty} n(t, m, a)=0$, ce qui signifie simplement, d'un point de vue biologique, que toutes les cellules sont condamnées à mourir.

En utilisant la méthode des caractéristiques nous obtenons

$$
p(t, m, a)=\left\{\begin{array}{l}
p\left(0, \pi_{-t}(m), a-t\right) \xi(m, t), \text { si } 0 \leq t<a \\
p\left(t-a, \pi_{-a}(m), 0\right) \xi(m, a), \text { si } t \geq a .
\end{array}\right.
$$

Nous pouvons alors écrire, en utilisant (13),

$$
\begin{aligned}
p(t, m, \bar{\tau}) & =p\left(0, \pi_{-t}(m), \bar{\tau}-t\right) \xi(m, t), \\
& =\Gamma\left(\pi_{-t}(m), \bar{\tau}-t\right) \xi(m, t),
\end{aligned}
$$

si $0 \leq t<\bar{\tau}$, et

$$
\begin{aligned}
p(t, m, \bar{\tau}) & =p\left(t-\bar{\tau}, \pi_{-\bar{\tau}}(m), 0\right) \xi(m, \bar{\tau}) \\
& =\beta\left(\pi_{-\bar{\tau}}(m), N\left(t-\bar{\tau}, \pi_{-\bar{\tau}}(m)\right)\right) N\left(t-\bar{\tau}, \pi_{-\bar{\tau}}(m)\right) \xi(m, \bar{\tau}),
\end{aligned}
$$

si $t \geq \bar{\tau}$. 
En utilisant la relation (6), nous obtenons bien que $P(t, m)$ vérifie les relations (11) et (12).

De même, d'après (5) et en utilisant (13), nous pouvons écrire :

$$
\begin{aligned}
n(t, m, 0) & =2 \int_{\underline{\tau}}^{\bar{\tau}} k(m, a) p\left(t, g^{-1}(m), a\right) d a \\
& =2 \int_{\underline{\tau}}^{\bar{\tau}} k(m, a) p(0, \Delta(t, m), a-t) \xi\left(g^{-1}(m), t\right) d a \\
& =2 \xi\left(g^{-1}(m), t\right) \int_{\underline{\tau}}^{\bar{\tau}} k(m, a) \Gamma(\Delta(t, m), a-t) d a
\end{aligned}
$$

si $0 \leq t<\underline{\tau}$, et

$$
\begin{aligned}
n(t, m, 0)= & 2 \int_{\underline{\tau}}^{t} k(m, a) p\left(t, g^{-1}(m), a\right) d a \\
& +2 \int_{t}^{\bar{\tau}} k(m, a) p\left(t, g^{-1}(m), a\right) d a \\
= & 2 \int_{\underline{\tau}}^{t} k(m, a) p(t-a, \Delta(a, m), 0) \xi\left(g^{-1}(m), a\right) d a \\
& +2 \int_{t}^{\bar{\tau}} k(m, a) p(0, \Delta(t, m), a-t) \xi\left(g^{-1}(m), t\right) d a, \\
= & 2 \int_{\underline{\tau}}^{t} k(m, a) \beta(\Delta(a, m), N(t-a, \Delta(a, m))) N(t-a, \Delta(a, m)) \times \\
& \xi\left(g^{-1}(m), a\right) d a+2 \xi\left(g^{-1}(m), t\right) \int_{t}^{\bar{\tau}} k(m, a) \Gamma(\Delta(t, m), a-t) d a,
\end{aligned}
$$

si $\underline{\tau} \leq t<\bar{\tau}$, et

$$
\begin{aligned}
n(t, m, 0)= & 2 \int_{\underline{\underline{\tau}}}^{\bar{\tau}} k(m, a) p(t-a, \Delta(a, m), 0) \xi\left(g^{-1}(m), a\right) d a, \\
= & 2 \int_{\underline{\tau}}^{\bar{\tau}} k(m, a) \beta(\Delta(a, m), N(t-a, \Delta(a, m))) \times \\
& N(t-a, \Delta(a, m)) \xi\left(g^{-1}(m), a\right) d a .
\end{aligned}
$$

si $t \geq \bar{\tau}$.

$\bar{N}(t, m)$ vérifie donc bien les équations (7), (8) et (9).

En remarquant que, pour tout $m \in[g(1), 1], \zeta(m, a)=0$ et $k(m, a)=0$, nous obtenons l'équation $(10)$.

Nous remarquons tout d'abord que les solutions des équations (7), (8) et (11) deviennent des conditions initiales pour les équations (9) et (12) respectivement. De plus, les solutions de l'équation (9) ne dépendent pas des solutions de l'équation (12). Enfin, si nous connaissons le comportement des solutions de (9) nous pouvons en déduire les solutions de l'équation (12) 
par la méthode des caractéristiques. Nous concentrerons donc notre étude sur les équations décrivant le comportement des cellules dans la phase de repos.

Nous allons déterminer une formulation faible du problème (7)-(8)-(9).

\section{Formulation faible}

Soit $\mathcal{C}^{0}[0, g(1)]$ l'espace des fonctions continues sur $[0, g(1)]$, muni de la norme du maximum, et soit $A: \mathcal{D}(A) \subset \mathcal{C}^{0}[0, g(1)] \rightarrow \mathcal{C}^{0}[0, g(1)]$ l'opérateur défini sur l'ensemble :

$$
\mathcal{D}(A)=\left\{u \in \mathcal{C}^{0}[0, g(1)] ; u \in \mathcal{C}^{1}(0, g(1)], \lim _{m \rightarrow 0} V(m) u^{\prime}(m)=0\right\}
$$

par

$$
A u(m)= \begin{cases}-\left(\delta(m)+V^{\prime}(m)\right) u(m)-V(m) u^{\prime}(m), & \text { si } m \in(0, g(1)] \\ -\left(\delta(0)+V^{\prime}(0)\right) u(0), & \text { si } m=0 .\end{cases}
$$

Proposition 4.1 A est le générateur infinitésimal du $C_{0}$-semi-groupe $(S(t))_{t \geq 0}$ défini, pour $\psi \in \mathcal{D}(A), m \in[0, g(1)]$ et $t \geq 0$, par :

$$
(S(t) \psi)(m)=\psi\left(h^{-1}\left(h(m) e^{-t}\right)\right) \exp \left\{-\int_{0}^{t}\left(\delta+V^{\prime}\right)\left(h^{-1}\left(h(m) e^{-s}\right)\right) d s\right\} .
$$

Remarque 4.1 Comme $h^{-1}\left(h(m) e^{s}\right)=\pi_{s}(m)$ pour tout $m \in[0,1]$ et tout $s \leq 0$, alors, $\forall \psi \in \mathcal{D}(A), \forall m \in[0, g(1)]$ et $\forall t \geq 0$ :

$$
(S(t) \psi)(m)=\psi\left(\pi_{-t}(m)\right) K(t, m)
$$

où

$$
K(t, m)=\exp \left\{-\int_{0}^{t} \delta\left(\pi_{-s}(m)\right)+V^{\prime}\left(\pi_{-s}(m)\right) d s\right\}
$$

pour tout $m \in[0, g(1)]$ et tout $t \geq 0$.

Preuve de la Proposition : La démonstration de cette proposition nécessite l'utilisation de trois lemmes.

Soit $B: \mathcal{D}(B) \subset \mathcal{C}^{0}[0, g(1)] \rightarrow \mathcal{C}^{0}[0, g(1)]$ l'opérateur défini par :

$$
\begin{gathered}
\mathcal{D}(B)=\left\{u \in \mathcal{C}^{0}[0, g(1)] ; u \in \mathcal{C}^{1}(0, g(1)], \lim _{m \rightarrow 0} V(m) u^{\prime}(m)=0\right\}, \\
B u(m)= \begin{cases}-V(m) u^{\prime}(m), & \text { si } m \in(0, g(1)], \\
0, & \text { si } m=0 .\end{cases}
\end{gathered}
$$

Lemme 4.1 L'opérateur $B$ défini par 1 (15) sur $\mathcal{D}(B)$ est le générateur infinitésimal d'un semi-groupe de contractions sur $\mathcal{C}^{0}[0, g(1)]$. 
Preuve : Nous allons utiliser le théorème de Hille-Yosida. $B$ est un opérateur linéaire fermé. De plus, en remarquant que $\mathcal{C}^{1}[0, g(1)] \subset \mathcal{D}(B) \subset \mathcal{C}^{0}[0, g(1)]$ et comme $\mathcal{C}^{1}[0, g(1)]$ est dense dans $\mathcal{C}^{0}[0, g(1)]$, nous obtenons bien que $\mathcal{D}(B)$ est dense dans $\mathcal{C}^{0}[0, g(1)]$.

Soient $w \in \mathcal{C}^{0}[0, g(1)]$ et $\lambda>0$ donnés. Montrons qu'il existe une fonction $u \in \mathcal{D}(B)$ et une seule telle que

$$
(I-\lambda B) u=w
$$

où $I$ est l'opérateur identité, et que

$$
|u|_{\infty} \leq|w|_{\infty}
$$

où la norme $|\cdot|_{\infty}$ est définie par

$$
|u|_{\infty}=\sup _{0 \leq m \leq g(1)}|u(m)| .
$$

Si $u$ existe alors $u$ est solution de l'équation différentielle

$$
\left\{\begin{array}{l}
u(m)+\lambda V(m) u^{\prime}(m)=w(m), \quad \text { pour } 0<m \leq g(1), \\
u(0)=w(0) .
\end{array}\right.
$$

Considérons la fonction $h_{\lambda}$ définie par

$$
h_{\lambda}(m)= \begin{cases}\exp \left(\int_{g(1)}^{m} \frac{d s}{\lambda V(s)}\right) & \text { pour } m \in(0, g(1)], \\ 0, & \text { pour } m=0 .\end{cases}
$$

Les solutions de l'équation (16) sont de la forme

$$
u(m)=\frac{1}{h_{\lambda}(m)}\left(k+\int_{g(1)}^{m} \frac{w(s)}{\lambda V(s)} h_{\lambda}(s) d s\right),
$$

pour $m \in(0, g(1)]$ et $k \in \mathbb{R}$. $h_{\lambda}$ est continue sur $[0, g(1)]$, de classe $\mathcal{C}^{1}$ sur $(0, g(1)]$ et

$$
h_{\lambda}^{\prime}(m)=\frac{h_{\lambda}(m)}{\lambda V(m)},
$$

pour $m \in(0, g(1)]$. Donc

$$
\int_{g(1)}^{m} \frac{h_{\lambda}(s)}{\lambda V(s)} d s=h_{\lambda}(m)-h_{\lambda}(g(1)) .
$$

Ainsi, $\lim _{m \rightarrow 0} \int_{g(1)}^{m} \frac{h_{\lambda}(s)}{\lambda V(s)} d s=-h_{\lambda}(g(1))=-1$. Par conséquent, $\frac{h_{\lambda}(s)}{\lambda V(s)} \in L^{1}(0, g(1))$ et, comme $h_{\lambda}(0)=0$, la fonction $u$ donnée par (17) est bornée si et seulement si

$$
k=\int_{0}^{g(1)} \frac{w(s)}{\lambda V(s)} h_{\lambda}(s) d s .
$$

L'unique solution possible de l'équation (16) est donc

$$
u(m)=\frac{1}{h_{\lambda}(m)}\left(\int_{0}^{m} \frac{w(s)}{\lambda V(s)} h_{\lambda}(s) d s\right),
$$


pour $m \in(0, g(1)]$, et $u(0)=w(0)$.

De plus, si $u \in \mathcal{D}(B)$, alors $|u(m)| \leq|w|_{\infty}$ pour tout $m \in(0, g(1)]$ et, comme $u(0)=w(0)$, $|u|_{\infty} \leq|w|_{\infty}$.

En appliquant le théorème de Hille-Yosida, nous achevons la démonstration du lemme.

Lemme 4.2 Si $\psi \in \mathcal{D}(B)$, alors

$$
\lim _{t \rightarrow 0^{+}} \frac{\psi\left(h^{-1}\left(h(m) e^{-t}\right)\right)-\psi(m)}{t}=-V(m) \psi^{\prime}(m) .
$$

Preuve : Nous effectuons le changement de variables suivant :

$$
y=h^{-1}\left(h(m) e^{-t}\right) .
$$

Alors, par définition de $h$,

$$
t=\ln \left(\frac{h(m)}{h(y)}\right)=\int_{y}^{m} \frac{d s}{V(s)}
$$

Ainsi

$$
\begin{aligned}
\lim _{t \rightarrow 0^{+}} \frac{\psi\left(h^{-1}\left(h(m) e^{-t}\right)\right)-\psi(m)}{t} & =\lim _{y \rightarrow m} \frac{\psi(y)-\psi(m)}{y-m} \frac{y-m}{\int_{y}^{m} \frac{d s}{V(s)}} \\
& =-V(m) \psi^{\prime}(m) .
\end{aligned}
$$

Ce qui conclut la démonstration.

Par définition du générateur infinitésimal d'un semi-groupe, nous déduisons de ce lemme que le semi-groupe engendré par $B$, que nous notons $(T(t))_{t \geq 0}$, est donné, pour tout $\psi \in \mathcal{D}(B)$ et tout $t \geq 0$, par

$$
(T(t) \psi)(m)=\psi\left(h^{-1}\left(h(m) e^{-t}\right)\right), \forall m \in[0, g(1)] .
$$

Lemme 4.3 Si $(T(t))_{t \geq 0}$ est le semi-groupe engendré par $B$ alors le semi-groupe engendré par $B+\alpha I$, avec $\alpha \in \mathcal{C}^{0}[0, g(1)]$, est défini par

$$
(S(t) \psi)(m)=\exp \left(\int_{0}^{t}(T(s) \alpha)(m) d s\right)(T(t) \psi)(m)
$$

pour tout $\psi \in \mathcal{D}(B)$, tout $m \in[0, g(1)]$ et tout $t \geq 0$.

Preuve : Il suffit de montrer que pour $\psi \in \mathcal{D}(B)$,

$$
\lim _{t \rightarrow 0^{+}} \frac{(S(t) \psi)(m)-\psi(m)}{t}=(B+\alpha I) \psi(m) .
$$


D'après la définition du semi-groupe $(S(t))$ nous avons :

$$
\begin{aligned}
\frac{(S(t) \psi)(m)-\psi(m)}{t} & =\frac{1}{t}\left[\exp \left(\int_{0}^{t}(T(s) \alpha)(m) d s\right)(T(t) \psi)(m)-\psi(m)\right] \\
& =\exp \left(\int_{0}^{t}(T(s) \alpha)(m) d s\right)\left(\frac{(T(t) \psi)(m)-\psi(m)}{t}\right) \\
& +\frac{1}{t}\left[\exp \left(\int_{0}^{t}(T(s) \alpha)(m) d s\right) \psi(m)-\psi(m)\right] .
\end{aligned}
$$

En passant à la limite sur $t$ nous obtenons le résultat : le premier terme de droite tend vers $B \psi(m)$ et le deuxième vers $(\alpha I) \psi(m)$.

En utilisant les lemmes 4.2 et 4.3 , avec $\alpha=\delta+V^{\prime}$, nous obtenons le résultat énoncé dans la proposition. $\square$.

Proposition 4.2 Soit $\varphi \in \mathcal{C}^{0}([0, \bar{\tau}] \times[0, g(1)])$. La formulation intégrée du problème (囚)(8)-(3) est :

$$
\left\{\begin{array}{llrl}
N(t, m) & =\varphi\left(\bar{\tau}, \pi_{-(t-\bar{\tau})}(m)\right) K(t-\bar{\tau}, m)+G(N)(t, m) \\
& -J(N)(t, m) & \text { si } t \geq \bar{\tau} \\
N(t, m) & =\varphi(t, m), & \text { si } 0 \leq t \leq \bar{\tau}
\end{array}\right.
$$

pour tout $m \in[0, g(1)]$, avec

$$
\begin{gathered}
G(N)(t, m)=2 \int_{\bar{\tau}}^{t}\left(\int _ { \underline { \underline { \tau } } } ^ { \overline { \tau } } \zeta ( \pi _ { - ( t - s ) } ( m ) , a ) \beta \left(\Delta\left(a, \pi_{-(t-s)}(m)\right), N\left(s-a, \Delta\left(a, \pi_{-(t-s)}(m)\right)\right) \times\right.\right. \\
\left.N\left(s-a, \Delta\left(a, \pi_{-(t-s)}(m)\right)\right) d a\right) K(t-s, m) d s,
\end{gathered}
$$

$e t$

$$
J(N)(t, m)=\int_{\bar{\tau}}^{t} K(t-s, m) \beta\left(\pi_{-(t-s)}(m), N\left(s, \pi_{-(t-s)}(m)\right)\right) N\left(s, \pi_{-(t-s)}(m)\right) d s
$$

pour tous $t \geq \bar{\tau}$ et $m \in[0, g(1)]$.

Preuve : L'équation (9) se met sous la forme :

$$
\begin{gathered}
\frac{\partial}{\partial t} N(t, m)=-\left(\delta(m)+\beta(m, N(t, m))+V^{\prime}(m)\right) N(t, m)-V(m) \frac{\partial}{\partial m} N(t, m) \\
+2 \int_{\underline{\tau}}^{\bar{\tau}} \zeta(m, a) \beta(\Delta(a, m), N(t-a, \Delta(a, m))) N(t-a, \Delta(a, m)) d a
\end{gathered}
$$

Soit $u$ l'application définie pour tout $t \geq 0$ par :

$$
u(t)(m)=N(t, m), \quad \forall m \in[0,1] .
$$


Comme

$$
N(t-a, \Delta(a, m))=u(t-a)(\Delta(a, m)):=u_{-a}(t)(\Delta(a, m)),
$$

il vient, d'après (19), pour tout $m \in[0,1]$ :

$$
\begin{aligned}
u^{\prime}(t)(m)= & -\left(\delta(m)+V^{\prime}(m)\right) u(t)(m)-V(m)(u(t))^{\prime}(m)-\beta(m, u(t)(m)) u(t)(m) \\
& +2 \int_{\underline{\tau}}^{\bar{\tau}} \zeta(m, a) \beta(\Delta(a, m), u(t-a)(\Delta(a, m))) u_{-a}(t)(\Delta(a, m)) d a,
\end{aligned}
$$

c'est-à-dire

$$
\begin{aligned}
u^{\prime}(t)(m)= & A u(t)(m)-\beta(m, u(t)(m)) u(t)(m) \\
& +2 \int_{\underline{\tau}}^{\bar{\tau}} \zeta(m, a) \beta(\Delta(a, m), u(t-a)(\Delta(a, m))) u_{-a}(t)(\Delta(a, m)) d a .
\end{aligned}
$$

$u(t)$ est donc donné, pour tout $m \in[0, g(1)]$, par la formule :

$$
\begin{aligned}
u(t)(m)= & S(t-\bar{\tau}) \varphi(\bar{\tau})(m)-\int_{\bar{\tau}}^{t} S(t-s) \beta\left(\pi_{-(t-s)}(m), u(s)\left(\pi_{-(t-s)}(m)\right)\right) \times \\
& u(s)\left(\pi_{-(t-s)}(m)\right) d s+2 \int_{\bar{\tau}}^{t} S(t-s)\left(\int_{\underline{\tau}}^{\bar{\tau}} \zeta(m, a) \times\right. \\
& \left.\beta(\Delta(a, m), u(t-a)(\Delta(a, m))) u_{-a}(s)(\Delta(a, m)) d a\right) d s,
\end{aligned}
$$

si $t \geq \bar{\tau}$, et $u(t)(m)=\varphi(t)(m):=\varphi(t, m)$ si $t \leq \bar{\tau}$, où $\varphi(t) \in \mathcal{C}^{0}([0, g(1)])$ pour tout $t \geq 0$.

D'après (14), si $t \geq \bar{\tau}$,

$$
\begin{aligned}
u(t)(m)= & \varphi\left(\bar{\tau}, \pi_{-(t-\bar{\tau})}(m)\right) K(t-\bar{\tau}, m)-\int_{\bar{\tau}}^{t} \beta\left(\pi_{-(t-s)}(m), u(s)\left(\pi_{-(t-s)}(m)\right)\right) \times \\
& u(s)\left(\pi_{-(t-s)}(m)\right) K(t-s, m) d s+2 \int_{\bar{\tau}}^{t}\left(\int_{\underline{\tau}}^{\bar{\tau}} \zeta\left(\pi_{-(t-s)}(m), a\right) \times\right. \\
& \left.\beta(\Delta(a, m), u(t-a)(\Delta(a, m))) u_{-a}(s)\left(\Delta\left(a, \pi_{-(t-s)}(m)\right)\right) d a\right) K(t-s, m) d s .
\end{aligned}
$$

En utilisant (20) nous obtenons alors (18).

\section{Existence et unicité}

Nous allons à présent établir l'existence et l'unicité des solutions du problème (18), en utilisant une méthode itérative et la méthode des pas, avant de montrer que l'unicité des solutions ne dépend en fait que des cellules de petites maturités.

Nous faisons l'hypothèse suivante :

$\left(\mathbf{H}_{1}\right)$ Il existe $l \in \mathbb{R}^{+}$tel que $\forall m \in[0, g(1)], \forall x_{1}, x_{2} \in \mathbb{R}^{+}$,

$$
\left|x_{1} \beta\left(m, x_{1}\right)-x_{2} \beta\left(m, x_{2}\right)\right| \leq l\left|x_{1}-x_{2}\right| .
$$


Théorème 5.1 Sous l'hypothèse $\left(\mathbf{H}_{1}\right)$, pour toute donnée initiale $\varphi \in \mathcal{C}^{0}([0, \bar{\tau}] \times[0, g(1)])$, le problème (18) possède une unique solution qui est continue.

Preuve : Soit $\varphi \in \mathcal{C}^{0}([0, \bar{\tau}] \times[0, g(1)])$ donnée. Si $N$ est solution du problème (18) alors

$$
N(t, m)=\varphi\left(\bar{\tau}, \pi_{-(t-\bar{\tau})}(m)\right) K(t-\bar{\tau}, m)+G(N)(t, m)-J(N)(t, m),
$$

pour tout $t \geq \bar{\tau}$ et tout $m \in[0, g(1)]$, et $N(t, m)=\varphi(t, m)$ pour $t \in[0, \bar{\tau}]$ et $m \in[0, g(1)]$.

Soit $t \in[0, \bar{\tau}+\underline{\tau}]$. Nous définissons la suite $\left(N_{n}\right)_{n \in \mathbb{N}}$ de la façon suivante :

si $t \in[0, \bar{\tau}]$ alors $N_{n}(t, m)=\varphi(t, m), \forall m \in[0, g(1)]$, et si $t \in[\bar{\tau}, \bar{\tau}+\underline{\tau}]$ alors, $\forall m \in[0, g(1)]$,

$$
\left\{\begin{array}{l}
N_{0}(t, m)=\varphi\left(\bar{\tau}, \pi_{-(t-\bar{\tau})}(m)\right) K(t-\bar{\tau}, m)+G(\varphi)(t, m) \\
N_{n}(t, m)=N_{0}(t, m)-J\left(N_{n-1}\right)(t, m)
\end{array}\right.
$$

Nous avons alors :

$$
\left|N_{1}(t, m)-N_{0}(t, m)\right|=\left|J\left(N_{0}\right)(t, m)\right| .
$$

Les fonctions $\delta$ et $V^{\prime}$ étant continues sur $[0, g(1)]$, l'application $K$ est continue sur $[0,+\infty) \times$ $[0, g(1)]$ et donc bornée sur $[\bar{\tau}, \bar{\tau}+\underline{\tau}] \times[0, g(1)]$. Notons

$$
\alpha=\max _{(t, m) \in[\bar{\tau}, \bar{\tau}+\underline{\tau}] \times[0, g(1)]}|K(t, m)| .
$$

Alors,

$$
\begin{aligned}
\left|N_{1}(t, m)-N_{0}(t, m)\right| & \leq \alpha \int_{\bar{\tau}}^{t}\left|\beta\left(\pi_{-(t-s)}(m), N_{0}\left(s, \pi_{-(t-s)}(m)\right)\right)\right| \times \\
& \leq \alpha l \int_{\bar{\tau}}^{t}\left|N_{0}\left(s, \pi_{-(t-s)}(m)\right)\right| d s,
\end{aligned}
$$

$\varphi, K$ et $G(\varphi)$ étant continues, $N_{0}$ est continue sur $[\bar{\tau}, \bar{\tau}+\underline{\tau}] \times[0, g(1)]$ :

$$
\exists M \geq 0, \forall m \in[0, g(1)], \forall t \in[\bar{\tau}, \bar{\tau}+\underline{\tau}],\left|N_{0}(t, m)\right| \leq M .
$$

Alors,

$$
\left|N_{1}(t, m)-N_{0}(t, m)\right| \leq M \alpha l(t-\bar{\tau})
$$

Montrons par récurrence le résultat suivant :

$$
\left(\mathbf{H}_{n}\right) \forall t \in[\bar{\tau}, \bar{\tau}+\underline{\tau}], \forall m \in[0, g(1)],\left|N_{n}(t, m)-N_{n-1}(t, m)\right| \leq M(\alpha l)^{n} \frac{(t-\bar{\tau})^{n}}{n !} .
$$

La relation est vraie pour $n=0$, nous venons de le vérifier. Supposons $\left(\mathbf{H}_{n}\right)$ satisfaite et montrons que $\left(\mathbf{H}_{n+1}\right)$ est réalisée.

Soient $t \in[\bar{\tau}, \bar{\tau}+\underline{\tau}]$ et $m \in[0, g(1)]$. Alors,

$$
\left|N_{n+1}(t, m)-N_{n}(t, m)\right|=\left|J\left(N_{n}\right)(t, m)-J\left(N_{n-1}\right)(t, m)\right|
$$


donc, en utilisant l'hypothèse $\left(\mathbf{H}_{1}\right)$,

$$
\begin{aligned}
\left|N_{n+1}(t, m)-N_{n}(t, m)\right| \leq \alpha l \int_{\bar{\tau}}^{t} \mid & N_{n}\left(s, \pi_{-(t-s)}(m)\right) \\
& -N_{n-1}\left(s, \pi_{-(t-s)}(m)\right) \mid d s .
\end{aligned}
$$

En utilisant l'hypothèse de récurrence, nous obtenons

$$
\begin{aligned}
\left|N_{n+1}(t, m)-N_{n}(t, m)\right| & \leq M(\alpha l)^{n+1} \int_{\bar{\tau}}^{t} \frac{(s-\bar{\tau})^{n}}{n !} d s \\
& \leq M(\alpha l)^{n+1} \frac{(t-\bar{\tau})^{n+1}}{(n+1) !}
\end{aligned}
$$

Nous obtenons bien le résultat escompté.

Par conséquent, $N:=\lim _{n \rightarrow \infty} N_{n}$ existe uniformément sur $[\bar{\tau}, \bar{\tau}+\underline{\tau}]$ et est continue.

Montrons que $N$ est solution de (18) pour $t \in[\bar{\tau}, \bar{\tau}+\underline{\tau}]$ et $m \in[0, g(1)]$, c'est-à-dire

$$
N(t, m)=N_{0}(t, m)-J(N)(t, m)
$$

car $G(N)(t, m)=G(\varphi)(t, m)$, pour tout $t \in[\bar{\tau}, \bar{\tau}+\underline{\tau}]$ et tout $m \in[0, g(1)]$. En effet, si $a \in[\underline{\tau}, \bar{\tau}]$ et $s \in[\bar{\tau}, t]$ alors $s-a \in[0, t-\underline{\tau}] \subset[0, \bar{\tau}]$ et donc

$$
N(s-a, \Delta(a, m))=\varphi(s-a, \Delta(a, m)),
$$

pour tout $m \in[0, g(1)]$. Posons

$$
v(t, m)=\left|N(t, m)-N_{0}(t, m)-J(N)(t, m)\right|,
$$

pour tout $t \in[\bar{\tau}, \bar{\tau}+\underline{\tau}]$ et tout $m \in[0, g(1)]$ et montrons que $v \equiv 0$.

En remarquant que

$$
N(t, m)-N_{n}(t, m)=\sum_{p=n}^{\infty}\left(N_{p+1}(t, m)-N_{p}(t, m)\right),
$$


alors

$$
\begin{aligned}
v(t, m) \leq & \left|N(t, m)-N_{n+1}(t, m)\right|+\left|J\left(N_{n}\right)(t, m)-J(N)(t, m)\right| \\
\leq & \left|N(t, m)-N_{n+1}(t, m)\right| \\
& \quad+\alpha l \int_{\bar{\tau}}^{t}\left|N_{n}\left(s, \pi_{-(t-s)}(m)\right)-N\left(s, \pi_{-(t-s)}(m)\right)\right| d s \\
\leq & \sum_{p=n+1}^{\infty}\left|N_{p+1}(t, m)-N_{p}(t, m)\right| \\
& +\alpha l \int_{\bar{\tau}}^{t} \sum_{p=n}^{\infty}\left|N_{p+1}\left(s, \pi_{-(t-s)}(m)\right)-N_{p}\left(s, \pi_{-(t-s)}(m)\right)\right| d s \\
\leq & M \sum_{p=n+1}^{\infty}(\alpha l)^{p+1} \frac{(t-\bar{\tau})^{p+1}}{(p+1) !}+M \alpha l \sum_{p=n}^{\infty} \int_{\bar{\tau}}^{t}(\alpha l)^{p+1} \frac{(s-\bar{\tau})^{p+1}}{(p+1) !} d s \\
\leq & M \sum_{p=n+1}^{\infty}(\alpha l)^{p+1} \frac{(t-\bar{\tau})^{p+1}}{(p+1) !}+M \sum_{p=n}^{\infty}(\alpha l)^{p+2} \frac{(t-\bar{\tau})^{p+2}}{(p+2) !} \\
\leq & 2 M \sum_{p=n+1}^{\infty}(\alpha l)^{p+1} \frac{(t-\bar{\tau})^{p+1}}{(p+1) !} .
\end{aligned}
$$

En faisant tendre $n$ vers $+\infty$, nous obtenons :

$$
v(t, m)=0, \quad \forall t \in[\bar{\tau}, \bar{\tau}+\underline{\tau}] \text { et } \forall m \in[0, g(1)] .
$$

Ainsi, $N(t, m)$ est solution de (18) pour $t \in[\bar{\tau}, \bar{\tau}+\underline{\tau}]$ et $m \in[0, g(1)]$.

Montrons que $N$ est unique.

Soit $Z(t, m)$ une autre solution de (18) $\operatorname{sur}[\bar{\tau}, \bar{\tau}+\underline{\tau}] \times[0, g(1)]$ :

$$
Z(t, m)=N_{0}(t, m)-J(Z)(t, m)
$$

pour tout $t \in[\bar{\tau}, \bar{\tau}+\underline{\tau}]$ et tout $m \in[0, g(1)]$.

Alors, $\forall t \in[\bar{\tau}, \bar{\tau}+\underline{\tau}]$ et $\forall m \in[0, g(1)]$,

$$
\begin{aligned}
|N(t, m)-Z(t, m)| & =|J(N)(t, m)-J(Z)(t, m)| \\
& \leq \alpha l \int_{\bar{\tau}}^{t}\left|N\left(s, \pi_{-(t-s)}(m)\right)-Z\left(s, \pi_{-(t-s)}(m)\right)\right| d s .
\end{aligned}
$$

Pour $t \in[\bar{\tau}, \bar{\tau}+\underline{\tau}]$ et $m \in[0, g(1)]$ nous posons :

$$
w(\sigma)=\left|N\left(\sigma, \pi_{-(t-\sigma)}(m)\right)-Z\left(\sigma, \pi_{-(t-\sigma)}(m)\right)\right|,
$$

pour tout $\sigma \in[\bar{\tau}, t]$.

Alors,

$$
\begin{gathered}
w(\sigma)=\left|J(N)\left(\sigma, \pi_{-(t-\sigma)}(m)\right)-J(Z)\left(\sigma, \pi_{-(t-\sigma)}(m)\right)\right|, \\
\leq \alpha l \int_{\bar{\tau}}^{\sigma} \mid N\left(s, \pi_{-(\sigma-s)} \circ \pi_{-(t-\sigma)}(m)\right) \\
\quad-Z\left(s, \pi_{-(\sigma-s)} \circ \pi_{-(t-\sigma)}(m)\right) \mid d r .
\end{gathered}
$$


Comme

$$
\pi_{-(\sigma-s)} \circ \pi_{-(t-\sigma)}=\pi_{-(t-s)},
$$

alors

$$
\begin{aligned}
w(\sigma) & \leq \alpha l \int_{\bar{\tau}}^{\sigma}\left|N\left(s, \pi_{-(t-s)}(m)\right)-Z\left(s, \pi_{-(t-s)}(m)\right)\right| d r \\
& \leq \alpha l \int_{\bar{\tau}}^{\sigma} w(s) d s .
\end{aligned}
$$

D'après le lemme de Gronwall, $\forall \sigma \in[\bar{\tau}, t], w(\sigma)=0$, donc $\forall \sigma \in[\bar{\tau}, \bar{\tau}+\underline{\tau}], w(\sigma)=0$. Ainsi, $\forall \sigma \in[\bar{\tau}, \bar{\tau}+\underline{\tau}], \forall m \in[0, g(1)]$,

$$
N\left(\sigma, \pi_{-(t-\sigma)}(m)\right)=Z\left(\sigma, \pi_{-(t-\sigma)}(m)\right) .
$$

Donc $N(t, m)=Z(t, m), \forall t \in[\bar{\tau}, \bar{\tau}+\underline{\tau}]$ et $\forall m \in[0, g(1)]$.

Le problème (18) possède donc une unique solution sur $[0, \bar{\tau}+\underline{\tau}] \times[0, g(1)]$ qui est continue. Notons $N^{*}$ cette solution.

En utilisant la suite $\left(N_{n}\right)_{n \in \mathbb{N}}$ définie de la façon suivante :

si $t \in[0, \bar{\tau}+\underline{\tau}]$ alors $N_{n}(t, m)=N^{*}(t, m), \forall m \in[0, g(1)]$, et si $t \in[\bar{\tau}+\underline{\tau}, \bar{\tau}+2 \underline{\tau}]$ alors, $\forall m \in[0, g(1)]$

$$
\left\{\begin{array}{l}
N_{0}(t, m)=\varphi\left(\bar{\tau}, \pi_{-(t-\bar{\tau})}(m)\right) K(t-\bar{\tau}, m)+G\left(N^{*}\right)(t, m), \\
N_{n}(t, m)=N_{0}(t, m)-J\left(N_{n-1}\right)(t, m)
\end{array}\right.
$$

nous montrons de la même manière que le problème (18) possède une unique solution, continue, sur $[0, \bar{\tau}+2 \underline{\tau}] \times[0, g(1)]$. Par la méthode des pas, l'existence et l'unicité d'une solution continue sur $[0,+\infty) \times[0, g(1)]$ sont établies.

Avant détablir le résultat d'unicité dépendant uniquement des cellules de petites maturités, nous montrons le résultat de positivité suivant.

Proposition 5.1 Nous supposons que $\delta(m)+V^{\prime}(m)>0$, pour tout $m \in[0, g(1)]$. Si, $\forall(t, m) \in[0, \bar{\tau}] \times[0, g(1)], N(t, m) \geq 0$, alors $N(t, m) \geq 0$, pour tout $t \geq \bar{\tau}$ et tout $m \in[0, g(1)]$.

Preuve : Nous donnons une idée de la démonstration. Nous utilisons la méthode des caractéristiques et la méthode des pas.

Nous notons $N=\varphi$ sur $[0, \bar{\tau}] \times[0, g(1)]$ et nous supposons $\varphi \geq 0$. Supposons alors qu'il existe $\left(t_{0}, m_{0}\right) \in[\bar{\tau}, \bar{\tau}+\underline{\tau}] \times[0, g(1)]$ tel que $N\left(t_{0}, m_{0}\right)<0$. Considérons alors l'équation différentielle

$$
\left\{\begin{array}{l}
m^{\prime}(t)=V(m(t)), \quad t \in[\bar{\tau}, \bar{\tau}+\underline{\tau}] \\
m\left(t_{0}\right)=m_{0}
\end{array}\right.
$$

et notons

$$
u(t)=N(t, m(t)), t \in[\bar{\tau}, \bar{\tau}+\underline{\tau}] .
$$

Alors

$$
u^{\prime}(t)=-\left(\delta(m(t))+V^{\prime}(m(t))+\beta(m(t), u(t))\right) u(t)
$$




$$
+2 \int_{\underline{\tau}}^{\bar{\tau}} \zeta(m(t), a) \beta(\Delta(a, m(t)), \varphi(t-a, \Delta(a, m(t)))) \varphi(t-a, \Delta(a, m(t))) d a .
$$

Donc $u^{\prime}\left(t_{0}\right)>0$. En effet, $u\left(t_{0}\right)<0$ et

$$
\int_{\underline{\tau}}^{\bar{\tau}} \zeta\left(m\left(t_{0}\right), a\right) \beta\left(\Delta\left(a, m\left(t_{0}\right)\right), \varphi\left(t_{0}-a, \Delta\left(a, m\left(t_{0}\right)\right)\right)\right) \varphi\left(t_{0}-a, \Delta\left(a, m\left(t_{0}\right)\right)\right) d a \geq 0 .
$$

Nous aboutissons à une contradiction. Donc $N(t, m) \geq 0$ sur $[\bar{\tau}, \bar{\tau}+\underline{\tau}] \times[0, g(1)]$. Par la méthode des pas, nous obtenons ce résultat pour $t \geq \bar{\tau}$. $\square$

Nous allons à présent établir un résultat qui souligne l'influence des cellules de petites maturités (cellules souches) sur l'évolution de la population totale de cellules.

Nous supposerons, dans la suite, qu'il existe $C \geq 0$ tel que pour tout $m \in(0, g(1)]$

$$
\int_{m}^{g^{-1}(m)} \frac{d s}{V(s)} \leq C .
$$

Exemple 5.1 Si $V(s) \underset{s \rightarrow 0}{\sim} \alpha s$, avec $\alpha>0$, alors

$$
\int_{m}^{g^{-1}(m)} \frac{d s}{V(s)} \leq C \text { si et seulement si } g^{\prime}(0)>0 \text {. }
$$

Théorème 5.2 Nous supposons l'hypothèse (22) vérifiée.

Soit $\tau_{0}=\sup _{m>0}\left(\int_{m}^{g^{-1}(m)} \frac{d s}{V(s)}\right)$. Supposons que $N_{1}(t, m)$ et $N_{2}(t, m)$ sont solutions de (18) pour des données initiales $\varphi_{1}$ et $\varphi_{2}$ respectivement. Supposons que $\underline{\tau}>\tau_{0}$ et qu'il existe $b \in\left(0, h^{-1}\left(e^{-\underline{\tau}}\right)\right)$ tel que

$$
\varphi_{1}(t, m)=\varphi_{2}(t, m), \quad \text { pour } m \in[0, b] \text { et } t \in[0, \bar{\tau}]
$$

Alors, il existe $\bar{t} \geq 0$ tel que

$$
N_{1}(t, m)=N_{2}(t, m), \quad \text { pour } m \in[0, g(1)] \text { et } t \geq \bar{t} .
$$

Preuve : Remarquons que la condition $\underline{\tau}>\tau_{0}$ implique, d'après le lemme 3.1, que $\Delta(a, m)<$ $m$ pour tout $m \in[0, g(1)]$ et tout $a \in[\underline{\tau}, \bar{\tau}]$, et que $h^{-1}\left(e^{-\underline{\tau}}\right)<g(1)$. De plus, comme $\underline{\tau}>0$, $h^{-1}\left(e^{-\underline{\tau}}\right)>0$.

Montrons tout d'abord que $N_{1}(t, m)=N_{2}(t, m)$, pour $m \in[0, b]$ et $t \geq 0$.

Soit $m \in[0, b]$. Si $t \in[0, \bar{\tau}]$, le résultat est évident.

Soit $t \geq \bar{\tau}$. Rappelons que si $N$ est solution du problème (18) alors

$$
N(t, m)=\varphi\left(\bar{\tau}, \pi_{-(t-\bar{\tau})}(m)\right) K(t-\bar{\tau}, m)+G(N)(t, m)-J(N)(t, m),
$$

pour tout $t \geq \bar{\tau}$ et tout $m \in[0, g(1)]$.

Comme $t-\bar{\tau} \geq 0$,

$$
\pi_{-(t-\bar{\tau})}(m)<m \leq b .
$$


Donc

$$
\varphi_{1}\left(\bar{\tau}, \pi_{-(t-\bar{\tau})}(m)\right)=\varphi_{2}\left(\bar{\tau}, \pi_{-(t-\bar{\tau})}(m)\right) .
$$

Soit $t \in[\bar{\tau}, \bar{\tau}+\underline{\tau}]$. Alors, $s-a \in[0, t-\underline{\tau}] \subset[0, \bar{\tau}]$ et

$$
\Delta\left(a, \pi_{-(t-s)}(m)\right)<\pi_{-(t-s)}(m)<m \leq b .
$$

Donc $G\left(N_{1}\right)(t, m)=G\left(N_{2}\right)(t, m)$. Par conséquent,

$$
\begin{aligned}
\left|N_{1}(t, m)-N_{2}(t, m)\right| & =\left|J\left(N_{1}\right)(t, m)-J\left(N_{2}\right)(t, m)\right| \\
& \leq \alpha \tilde{l} \int_{\bar{\tau}}^{t}\left|N_{1}\left(s, \pi_{-(t-s)}(m)\right)-N_{2}\left(s, \pi_{-(t-s)}(m)\right)\right| d s .
\end{aligned}
$$

Par un raisonnement analogue à celui présenté dans la démonstration précédente et en utilisant le lemme de Gronwall nous obtenons

$$
N_{1}(t, m)=N_{2}(t, m)
$$

pour tout $t \in[\bar{\tau}, \bar{\tau}+\underline{\tau}]$ et tout $m \in[0, b]$. Par la méthode des pas, nous obtenons le résultat pour $t \geq 0$.

Soit $\alpha$ l'application définie de $[0, g(1)]$ dans $[0, g(1)]$ par

$$
\alpha(m)=\Delta(\underline{\tau}, m) .
$$

D'après le lemme 3.1, $\alpha$ est une application croissante, atteignant son maximum en $g(1)$ et vérifiant $\alpha(m)<m$ pour tout $m \in(0, g(1)]$. $\alpha$ est donc inversible et nous notons $\Lambda$ son inverse. Comme $\alpha(g(1))=h^{-1}\left(e^{-\tau}\right), \Lambda$ est définie sur $\left[0, h^{-1}\left(e^{-\tau}\right)\right]$. Nous pouvons prolonger $\Lambda$ par continuité sur $\left[h^{-1}\left(e^{-\underline{\tau}}\right), g(1)\right]$ en posant

$$
\Lambda(m)=g(1), \text { pour tout } m \in\left[h^{-1}\left(e^{-\underline{\tau}}\right), g(1)\right] .
$$

$\alpha$ étant strictement croissante et positive sur $[0, g(1)], \Lambda$ est strictement croissante sur $\left[0, h^{-1}\left(e^{-\underline{\tau}}\right)\right]$. Considérons la suite $\left(b_{n}\right)_{n \in \mathbb{N}}$ définie par

$$
\left\{\begin{aligned}
b_{n+1} & =\Lambda\left(b_{n}\right), \text { pour } n \in \mathbb{N}, \\
b_{0} & =b .
\end{aligned}\right.
$$

La suite $\left(b_{n}\right)_{n \in \mathbb{N}}$ est croissante; en effet, $\forall n \in \mathbb{N}$,

$$
b_{n}=\alpha\left(b_{n+1}\right)=\Delta\left(\underline{\tau}, b_{n+1}\right)<b_{n+1} .
$$

Nous définissons aussi la suite $\left(t_{n}\right)_{n \in \mathbb{N}}$ par

$$
t_{n}=\ln \left(\frac{h\left(b_{n}\right)}{h(b)}\right)+n \bar{\tau},
$$

c'est-à-dire

$$
\left\{\begin{aligned}
t_{n+1} & =t_{n}+\bar{\tau}+\ln \left(\frac{h\left(b_{n+1}\right)}{h\left(b_{n}\right)}\right), \text { pour } n \in \mathbb{N} \\
t_{0} & =0
\end{aligned}\right.
$$


La suite $\left(t_{n}\right)_{n \in \mathbb{N}}$ est croissante.

Montrons par récurrence que

$$
\left(H_{n}\right) \quad \text { Si } m \in\left[0, b_{n}\right] \text { et } t \geq t_{n} \text { alors } N_{1}(t, m)=N_{2}(t, m) .
$$

Le résultat est vrai pour $n=0$, nous venons de le montrer.

Supposons $\left(H_{n}\right)$ vérifiée. Soient $m \in\left[0, b_{n+1}\right]$ et $t \geq t_{n+1}$. Si $t \geq t_{n+1}$ alors $t \geq t_{n}+\bar{\tau}$. Nous pouvons réécrire les solutions $N_{i}, i=1,2$, du problème (18) de la façon suivante :

$$
\begin{aligned}
N_{i}(t, m)= & N_{i}\left(t_{n}+\bar{\tau}, \pi_{-}\left(t-t_{n}-\bar{\tau}\right)\right. \\
+ & 2 \int_{t_{n}+\bar{\tau}}^{t}\left(\int_{\underline{\tau}}^{\bar{\tau}} \zeta\left(\pi_{-(t-s)}(m), a\right) N_{i}\left(s-a, \Delta\left(a, \pi_{-(t-s)}(m)\right)\right) \times\right. \\
& \left.\beta\left(\Delta\left(a, \pi_{-(t-s)}(m)\right), N_{i}\left(s-a, \Delta\left(a, \pi_{-(t-s)}(m)\right)\right)\right) d a\right) K(t-s, m) d s,
\end{aligned}
$$

pour $t \geq t_{n}+\bar{\tau}$.

Montrons que $N_{1}\left(t_{n}+\bar{\tau}, \pi_{-\left(t-t_{n}-\bar{\tau}\right)}(m)\right)=N_{2}\left(t_{n}+\bar{\tau}, \pi_{-\left(t-t_{n}-\bar{\tau}\right)}(m)\right)$.

$t \geq t_{n+1}$ donc

$$
t-t_{n}-\bar{\tau} \geq \ln \left(\frac{h\left(b_{n+1}\right)}{h\left(b_{n}\right)}\right)
$$

ou encore,

$$
e^{-\left(t-t_{n}-\bar{\tau}\right)} \leq \frac{h\left(b_{n}\right)}{h\left(b_{n+1}\right)}
$$

D'où

$$
h(m) e^{-\left(t-t_{n}-\bar{\tau}\right)} \leq h(m) \frac{h\left(b_{n}\right)}{h\left(b_{n+1}\right)} \leq h\left(b_{n+1}\right) \frac{h\left(b_{n}\right)}{h\left(b_{n+1}\right)}=h\left(b_{n}\right),
$$

car $h$ est croissante. Ainsi,

$$
\pi_{-\left(t-t_{n}-\bar{\tau}\right)}(m)=h^{-1}\left(h(m) e^{-\left(t-t_{n}-\bar{\tau}\right)}\right) \leq b_{n} .
$$

En utilisant l'hypothèse de récurrence, nous obtenons le résultat souhaité.

Enfin, comme $s-a \geq t_{n}$ et

$$
\Delta\left(a, \pi_{-(t-s)}(m)\right) \leq \Delta(a, m) \leq \Delta(\underline{\tau}, m) \leq \Delta\left(\underline{\tau}, b_{n+1}\right)=b_{n},
$$

nous obtenons

$$
\left|N_{1}(t, m)-N_{2}(t, m)\right|=\left|J\left(N_{1}\right)(t, m)-J\left(N_{2}\right)(t, m)\right| .
$$

Par un raisonnement analogue au précédent, utilisant le lemme de Gronwall, nous vérifions l'hypothèse $\left(H_{n+1}\right)$.

Remarquons que, par définition de $\Lambda$, il existe $M \in \mathbb{N}^{*}$ tel que

$$
b_{M}<g(1)=b_{M+1} .
$$

Alors, pour tout $m \in\left[0, b_{M+1}\right]=[0, g(1)]$ et tout $t \geq t_{M+1}$,

$$
N_{1}(t, m)=N_{2}(t, m) \text {. }
$$

Nous pouvons poser $\bar{t}=t_{M+1}$ et le théorème est alors démontré.

Nous déduisons de ce théorème le corollaire suivant : 
Corollaire 5.1 Nous supposons l'hypothèse (29) vérifiée.

Soit $\tau_{0}=\sup _{m>0}\left(\int_{m}^{g^{-1}(m)} \frac{d s}{V(s)}\right)$. Soit $\varphi \in \mathcal{C}^{0}([0, \bar{\tau}] \times[0, g(1)])$ et $N^{\varphi}$ la solution du problème (18) associée à la donnée initiale $\varphi$. Supposons que $\underline{\tau}>\tau_{0}$ et qu'il existe $b \in\left(0, h^{-1}\left(e^{-\tau}\right)\right)$ tel que $\varphi(t, m)=0$, pour $m \in[0, b]$ et $t \in[0, \bar{\tau}]$. Alors, il existe $\bar{t} \geq 0$ tel que $N^{\varphi}(t, m)=0$, pour $m \in[0, g(1)]$ et $t \geq \bar{t}$.

Ce résultat met en avant l'influence des cellules de petites maturités (cellules souches) sur la population future de cellules. Nous remarquons que dans le cas d'une absence de cellules souches dans le milieu initial (ce qui est le cas d'une anémie aplasique), l'extinction de la population après un temps fini $\bar{t}$ est inévitable, les cellules ne parviennent pas à survivre.

\section{Invariance - Application du théorème 5.2}

Dans toute cette partie, nous notons $N^{\varphi}$ la solution du problème (18) pour une donnée initiale $\varphi \in \mathcal{C}^{0}([0, \bar{\tau}] \times[0, g(1)])$. Pour tout réel $b \in(0, g(1)]$, nous définissons $\|.\|_{b}$ par

$$
\forall u \in \mathcal{C}^{0}([0, \bar{\tau}] \times[0, g(1)]), \quad\|u\|_{b}:=\sup \{|u(t, m)| ;(t, m) \in[0, \bar{\tau}] \times[0, b]\} .
$$

Nous supposons que

$$
I:=\inf _{m \in[0, g(1)]}\left(\delta(m)+V^{\prime}(m)\right)>0
$$

et nous notons

$$
\tilde{\zeta}:=\sup \{|\zeta(m, a)| ;(m, a) \in[0, g(1)] \times[0, \bar{\tau}]\} .
$$

Proposition 6.1 Supposons que $l(2(\bar{\tau}-\underline{\tau}) \tilde{\zeta}+1)<I$. Alors, pour tout $b \in(0, g(1)]$ et toute fonction $\varphi \in \mathcal{C}^{0}([0, \bar{\tau}] \times[0, g(1)])$,

$$
\forall t \geq \bar{\tau}, \quad \forall m \in[0, b], \quad\left|N^{\varphi}(t, m)\right| \leq\|\varphi\|_{b} .
$$

Preuve : Soient $b \in(0, g(1)]$ et $\varphi \in \mathcal{C}^{0}([0, \bar{\tau}] \times[0, g(1)])$. Pour $t \geq 0$ et $m \in[0, b]$, nous définissons la suite $\left(N_{n}\right)_{n \in \mathbb{N}}$ de la façon suivante $: \forall n \in \mathbb{N}, N_{n}(t, m)=\varphi(t, m)$ si $t \in[0, \bar{\tau}]$, et, si $t \geq \bar{\tau}$,

$$
\left\{\begin{array}{l}
N_{0}(t, m)=\varphi\left(\bar{\tau}, \pi_{-(t-\bar{\tau})}(m)\right) K(t-\bar{\tau}, m) \\
N_{n}(t, m)=N_{0}(t, m)+G\left(N_{n-1}\right)(t, m)-J\left(N_{n-1}\right)(t, m), n \geq 1 .
\end{array}\right.
$$

Alors, $\forall t \geq \bar{\tau}, \forall m \in[0, b]$,

$$
\left|N_{0}(t, m)\right| \leq e^{-I(t-\bar{\tau})}\|\varphi\|_{b} \leq\|\varphi\|_{b}
$$

Montrons, par récurrence, que, pour tout $t \geq \bar{\tau}$ et tout $m \in[0, b]$,

$$
\forall n \in \mathbb{N},\left|N_{n}(t, m)\right| \leq\|\varphi\|_{b}
$$

Le résultat est vrai pour $n=0$. Supposons qu'il est vrai au rang $n, n \geq 0$. Alors, pour tout $t \geq \bar{\tau}$ et tout $m \in[0, b]$

$$
\left|N_{n+1}(t, m)\right| \leq\left|N_{0}(t, m)\right|+\left|G\left(N_{n}\right)(t, m)\right|+\left|J\left(N_{n}\right)(t, m)\right| .
$$


Or,

$$
\left|G\left(N_{n}\right)(t, m)\right| \leq 2 l(\bar{\tau}-\underline{\tau}) \tilde{\zeta}\|\varphi\|_{b} \int_{\bar{\tau}}^{t} e^{-I(t-s)} d s
$$

et

$$
\left|J\left(N_{n}\right)(t, m)\right| \leq l\|\varphi\|_{b} \int_{\bar{\tau}}^{t} e^{-I(t-s)} d s
$$

Donc

$$
\left|N_{n+1}(t, m)\right| \leq\|\varphi\|_{b}\left(e^{-I(t-\bar{\tau})}+l(2(\bar{\tau}-\underline{\tau}) \tilde{\zeta}+1) \int_{\bar{\tau}}^{t} e^{-I(t-s)} d s\right) .
$$

En notant $\mu=\frac{l(2(\bar{\tau}-\underline{\tau}) \tilde{\zeta}+1)}{I}$, nous obtenons

$$
\left|N_{n+1}(t, m)\right| \leq\|\varphi\|_{b}\left(e^{-I(t-\bar{\tau})}+\mu\left(1-e^{-I(t-\bar{\tau})}\right)\right) .
$$

Comme $\mu<1$ par hypothèse, nous aboutissons à

$$
\left|N_{n+1}(t, m)\right| \leq\|\varphi\|_{b}
$$

Nous obtenons le résultat attendu.

D'après la démonstration du théorème 5.1, la suite $\left(N_{n}\right)_{n \in \mathbb{N}}$ converge vers $N$ lorsque $n$ tends vers $+\infty$. Par passage à la limite sur $\mathrm{n}$ nous obtenons finalement

$$
\left|N^{\varphi}(t, m)\right| \leq\|\varphi\|_{b}, \forall t \geq \bar{\tau}, \forall m \in[0, b] .
$$

La proposition est ainsi démontrée.

Nous déduisons de ce résultat, le théorème suivant.

Théorème 6.1 Si $l(2(\bar{\tau}-\underline{\tau}) \tilde{\zeta}+1)<I$ alors, pour tout $b \in(0, g(1)]$ et toute fonction $\varphi \in \mathcal{C}^{0}([0, \bar{\tau}] \times[0, g(1)])$, il existe $\bar{t} \geq 0$ tel que

$$
\forall t \geq \bar{t}, \quad \forall m \in[0, g(1)], \quad\left|N^{\varphi}(t, m)\right| \leq\|\varphi\|_{b} .
$$

Preuve : Soient $b \in(0, g(1)]$ et $\varphi \in C([0, \bar{\tau}] \times[0, g(1)])$. Nous définissons, pour $t \in[0, \bar{\tau}]$, l'application $\tilde{\varphi}$ par :

$$
\tilde{\varphi}(t, m)= \begin{cases}\varphi(t, m), & \text { si } m \in[0, b], \\ \varphi(t, b), & \text { si } m \in[b, g(1)] .\end{cases}
$$

D'après la proposition 6.1, pour tout $t \geq \bar{\tau}$ et tout $m \in[0, g(1)],\left|N^{\tilde{\varphi}}(t, m)\right| \leq\|\tilde{\varphi}\|_{g(1)}$. De plus, comme $\varphi(t, m)=\tilde{\varphi}(t, m)$ sur $[0, \bar{\tau}] \times[0, b]$, d'après le théorème 5.2 il existe $\bar{t} \geq 0$ tel que $N^{\varphi}(t, m)=N^{\tilde{\varphi}}(t, m)$ pour $t \geq \bar{\tau}$ et $m \in[0, g(1)]$. Enfin, comme $\|\tilde{\varphi}\|_{g(1)}=\|\varphi\|_{b}$, nous obtenons

$$
\forall t \geq \bar{t}, \quad \forall m \in[0, g(1)], \quad\left|N^{\varphi}(t, m)\right| \leq\|\varphi\|_{b}
$$

Ce théorème met en avant le résultat suivant : si l'inégalité $l(2(\bar{\tau}-\underline{\tau}) \tilde{\zeta}+1)<I$ est vérifiée, alors la population de cellules souches domine la population totale, au moins à partir d'un 
certain temps. Or l'inégalité $l(2(\bar{\tau}-\underline{\tau}) \tilde{\zeta}+1)<I$ est vérifiée, par exemple, si $l$ est petit ou $I$ grand, c'est-à-dire, biologiquement, si peu de cellules sont introduites de la phase de repos vers la phase de prolifération ( $\operatorname{car} l \geq \beta(m, x)$ pour tous $m \in[0, g(1)]$ et $x \geq 0$ ) ou si le taux de mortalité dans la phase de repos est élevé.

\section{Références}

[1] M. Adimy, L. Pujo-Menjouet, A singular transport model describing cellular division, C. R. Acad. Sci. Paris 332 série I, 1071-1076, 2001.

[2] J. Dyson, R. Villella-Bressan et G.F. Webb, A singular transport equation modelling a proliferating maturity structured cell population, Can. Appl. Math. Quart., 4, 65-95, 1996.

[3] L.L. Henry, Population analysis and models, Edward Arnold, London, 1976.

[4] N. Keyfitz, Introduction to the mathematics of population, Addison-Wesley, Reading, Mass, 1968.

[5] M.C. Mackey, Unified hypothesis of the origin of aplastic anaemia and periodic hematopoiesis, Blood 51, 941-956, 1978.

[6] M.C. Mackey et P. Dörmer, continuous maturation of proliferating erythroid precursors, Cell Tissue Kinet., 15, 381-392, 1982.

[7] M.C. Mackey et R. Rudnicki, Global stability in a delayed partial differential equation describing cellular replication, J. Math. Biol., 33, 89-109, 1994.

[8] J.H. Pollard, Mathematical models for the growth of human populations, Cambridge University Press, cambridge, 1973.

[9] L. Pujo-Menjouet, Contribution à l'étude d'une équation de transport à retards décrivant une dynamique de population cellulaire, Thèse de doctorat à Pau, Université de Pau et des Pays de l'Adour, 2001.

[10] A. Rey et M.C. Mackey, Bifurcations and travelling waves in a delayed partial differential equation, Chaos, 2, 231-244, 1992.

[11] A. Rey et M.C. Mackey, Multistability and boundary layer development in a transport equation with retarded arguments, Can. Appl. Math. Quart., 1, 1-21, 1993. 